\title{
Extension Modeling Strategy of Intelligent Detection in D.huoshanense Photosynthesis Process
}

\author{
Rongde Lu' ${ }^{1}$, Can Qin ${ }^{2}$, Yunsheng Bao ${ }^{2}$ \\ ${ }^{1}$ Teaching Center of Physical Experiments, Physics School, University of \\ Science \& Technology of China, Hefei, China \\ ${ }^{2}$ Department of Materials of Science and Engineering, University of \\ Science \& Technology of China, Hefei, China \\ E-mail:lrd@ustc.edu.cn, \{limbo,yongsbao\}@mail.ustc.edu.cn \\ Received December 9, 2010; revised January 16, 2011; accepted May 7, 2011
}

\begin{abstract}
Aiming at the limitations of the existing knowledge representations in intelligent detection, a new method of Extension-based Knowledge Representation (EKR) was proposed. The definitions, grammar rules, and storage structure of EKR were presented. An Extension Solving Model (ESM) based on EKR was discussed in detail, including creation of the extension constraint graph, extended inference, calculation of relevant functions and generation of extension set. A knowledge base system based on EKR and ESM was developed, which was applied in extension repository system intelligent design of detection in photosynthesis process of D.huoshanense. More reasonable results were obtained than traditional rule-based system. EKR was feasible in intelligent design to solve the problem of intelligent detection knowledge representations.
\end{abstract}

Keywords: Extension-Based Knowledge Representation (EKR), Intelligent Detection, Extension Modeling Strategy (EMS), Photosynthesis Process of D.huoshanense (PPDH)

\section{Introduction}

About the formation of creative thinking and the inherent regularity of the formation, it is a long period of exploration in human social practice. From social science to natural science, people are trying to find a description form, but it comes down to a burst of inspiration, or "The patent of genius" at end. Extenics [1] researches the laws and the methods of tings exploiting from things extension nature. As crossing academics, Extenics [1] lies in philosophy, thinking sciences and mathematics, and is used to solve a large number of contradictions and pseudo-contradictions existing in real world. Extension analysis breaks through old intuition or inspiration mode of discovery creative work and establishes formalization road to new discoveries, new inventions. Extension analysis makes ordinary people complete creative found work which used to be done only the wise.

Extension Modeling Strategy (EMS) uses to generate and exploit strategy set in modeling photosynthesis process of D.huoshanense, be able to provide adequate options for develop the correct modeling strategy. It can exploit more new programs to form the optimum strategy to solve practical problems when existing programs can not meet the requirements. The object of D.huoshanense photosynthesis process researched by EMS is variability, so the use of change and development perspectives to research practical issues is involved. Analyzing the object of D.huoshanense photosynthesis process in a dynamic system whose time, space and other factors change always, generates many programs, which is the basic form of the optimum modeling strategy, and this can not be seen directly. EKR [2] combines qualitative analysis and quantitative calculation. According to the extension of matter element when conflict between achieve the objective and constraints, or conflict during number of strategic objectives, using divergent, conjugation, relativity, implication and scalability of matter element exploits generation strategy set in extension modeling decision-making process. In a broader time and space to consider strategies of managing conflict, and through quantitatively calculation of the matter-element transformation and matter-element theory to improve strategies in accuracy and operation, EMS [3] does not exclude other strategy approaches and has a preferable compatibility with a variety of qualitative or quantitative 
strategy techniques. When meeting contradictions in decision process of EMS, EMS will play its unique advantages, combined with other strategies, in order to generate the best strategy. The part uses EMS to solve incompatible problem of control strategies at different stages of D.huoshanense photosynthesis process and the ill-posed problem of gaining empirical experience.

\section{Extension-Based Knowledge Representation}

\subsection{Divergence Method Generated by Modeling Strategy}

Establish the matter-element of decision objectives and restrict constraints $\{P\},\{p\}$.

According to the divergence of matter element (a matter with multi-feature, one feature with more matter, one value with more matter) [1], objective matter element is developed using transformation $T=T_{c} \vee T_{N} \vee T_{(N, c)}$ as follows.

$$
\begin{aligned}
P= & P \dashv\left\{\left(N_{i}, c_{i}, v_{i}\right) \mid i=1,2, \cdots, q\right\} \\
& \cup\left\{\left(N_{j}, c_{j}, v_{j}\right) \mid j=1,2, \cdots, q\right\} \\
= & W_{1}(P) \cup W_{2}(P) \cup W_{3}(P) \cdots
\end{aligned}
$$

According to the matter-element conjugation (imaginary-real, hard-soft, appearance-latent, positive-negative), with transformation $T=T_{g}$ analyses of material, systematic, dynamic and opposite of objective matter element is:

$$
\mathrm{P}-\left\{P_{i m}, P_{r e}, P_{s f}, P_{h r}, P_{l t}, P_{a p}, P_{n g}, P_{p c}\right\}=W_{4}(P)
$$

Analysis method of matter-element relevance and implication:

According to the scalability (additive, integral, divisibility) of matter-element, using transformation $T=T_{\otimes} \vee T_{\odot} \vee T_{/} \vee T_{/ /}$to integrate conditions matterelement $r=\left(N^{\prime}, c^{\prime}, v^{\prime}\right)$ :

$$
\begin{aligned}
& \mathrm{p}-\left\{p_{+}\right\} \cup\left\{p_{\times}\right\} \cup\left\{p_{/}\right\} \cup\left\{p_{/ /}\right\} \\
& =W_{\oplus}(p) \cup W_{\odot}(p) \cup W_{/}(p) \cup W_{/ /}(p)
\end{aligned}
$$

Find all the matter-element or the matter element subset contain $p$ in the matter-element set $W_{1}(P) \cup W_{2}(P) \cup$ $W_{3}(P) \cup W_{4}(P)$ constitute $W^{*}(P)$; Find all the matter element or the matter element subset contain $p$ in the matter-element set $W_{\oplus}(p) \cup W_{\odot}(p) \cup W_{/}(p) \cup W_{/ /}(p)$ or the matter element subset can take place of $P$ constitute $W^{*}(P)$, select the element in $W^{*}(P)$ to be able to satisfy some element request in $W^{*}(p)$ to constitute $W(P)$, corresponding element in $W^{*}(p)$ constitutes $W(p)$, this process can be described by following transformation [1]:

$$
\begin{aligned}
& \left\{\begin{array}{l}
T_{P}=\left\{T_{c}, T_{N}, T_{(N, c)}, T_{g}\right\} ; \\
T_{p}=\left\{T_{\oplus}, T_{\odot}, T_{/}, T_{/ /}\right\} ; \\
T_{P}^{*}\left\{W_{1}(P) \cup W_{2}(P) \cup W_{3}(P) \cup W_{4}(P)\right\}=W^{*}(P) ; \\
T_{p}^{*}=\left\{W_{\oplus}(p) \cup W_{\odot}(p) \cup W_{/}(p) \cup W_{/ /}(p)\right\}=W^{*}(p) ;
\end{array}\right. \\
& \left\{\begin{array}{l}
Y_{i}\left(R^{*}\right)=R^{\prime}, R^{*}: W^{*}(R), R^{\prime}: W(R), i=1,2, \cdots, k ; \\
T_{i}\left(r^{*}\right), r^{*}: W^{*}(r), r^{\prime}: W(r), i=1,2, \cdots, k ; \\
T=\left\{T_{i} \mid T_{i}=Y_{i} T_{R}^{*} T_{R} \wedge T_{i} T_{r}^{*} T_{r}, i=1,2, \cdots, k\right\}, R^{\prime} \downarrow\left(r^{\prime}\right)
\end{array}\right.
\end{aligned}
$$

If $W_{1}(R) \cup W_{2}(R) \cup W_{3}(R) \cup W_{4}(R)$ and $W_{\otimes}(r) \cup$ $W_{\odot}(r) \cup W_{/}(r) \cup W_{/ /}(r)$ contain a large number of matter elements, then search of $W^{*}(R), W^{*}(r)$ and the $W(R)$ may be completed by the computer.

According to the need of EMS, we still can extend modeling goals R by matter-element scalability, and extend condition $\mathrm{r}$ through the matter-element divergent, feasibility, relevance and implication. It can make content of $W(R)$ and $W(r)$ richer, and forms more choices of strategy, which used to solve the contradictions and pseudo-contradictions in EMS. Below discusses the application of ill-posed problem which was found by empirical experience in D.huoshanense photosynthesis system based on matter-element conjugate strategy in detail. The matter-element conjugated system is a kind of relatively closed system. The commonly met problem is how to transform a non-coordinated matter-element conjugate system into coordinated matter-element conjugate system. In this section we would solve the method of this problem, and apply it to the comprehensive modeling in D.huoshanense photosynthesis system, to produce successful conjugate strategy.

\subsection{The Matter-Element Conjugation and Conjugate Transformation}

The matter-element conjugation scoring structural properties of the matter element, it understands and analyzes things $N$ in material, systematic, dynamic and intrinsic opposition and unity from different angles. The matterelement conjugate structure presents four forms: imaginary-real, hard-soft, appearance-latent, positive-negative.

Expression for imaginary-real: $N=\operatorname{reN} \oplus i m N$, where reN means the real part of $N$, while $\operatorname{im} N$ means the imaginary part of $N$;

Expression for hard-soft: $N=h r N$ sf $N$, where $h r N$ and $s f N$ denote the hard part of $N$ and the soft part of $N$ respectively;

Expression for appearance-latent: $N=$ ap $N \oplus$ lt $N$, where ap $N$ and $l t N$ correspondingly denote the appear- 
ance part and the latent part of $N$;

Expression for positive-negative: $N=p s(c) N \oplus n g(c) N$, with $p s(c)$ and $N \oplus n g(c) N$ representing the positive part of $N$ (about characteristic $c$ ) and the negative part of $N(c)$ correspondingly.

When the matter-element transformation $\varphi$ acts on certain part of the thing $N($ e.g. $\varphi(h r N))$ which has the aforementioned structure, it often leads to another part of the $N($ e.g. $s f N)$ change, and leads to the change of the whole $N$. Here the transformation $\varphi$ is an initiative transformation, the transformation caused by $\varphi$ is conduction transformation, as $T_{\varphi}$. Such conduction transformation happens in tings conjugate department called conjugation transformation [4].

\subsection{Matter-Element Conjugation System and ITS Classification}

Suppose relative closed system $\Sigma$ with only two elements $N_{i}(i=1,2)$, and $N_{1} / / N_{2}, L\left(N_{1}, N_{2}\right)$ is external connection relation. Under the $L\left(N_{1}, N_{2}\right)$ function, if $N_{1}$ and $N_{2}$ are interdependent relations, we call $\Sigma$ for a matter-element conjugate system, as $\sum=N_{1} \odot L\left(N_{1}, N_{2}\right) \odot N_{2}$.

Property 1:

$$
N_{1} \odot L\left(N_{1}, N_{2}\right) \odot N_{2}=N_{2} \odot L\left(N_{1}, N_{2}\right) \odot N_{1}
$$

Now, if regard $\sum$ as a thing, the hard part of $\Sigma$ is $h r \sum=\left\{N_{1}, N_{2}\right\}$, and the soft part of $\sum$ is sf $\sum=L\left\{N_{1}, N_{2}\right\}$. Hence, the structural relationship:

Property 2:

$$
\sum=h r \sum \oplus s f \sum=\left\{N_{1}, N_{2}\right\} \oplus L\left(N_{1}, N_{2}\right)
$$

For ting $N_{i}(i=1,2)$, examine the matter-element $R_{i}=$ $\left(N_{i}, c_{i}, v_{i}\right)(i=1,2)$, and the extension relation of matter element:

$$
\begin{gathered}
A(W)=\left\{\left(R_{1}, R_{2}, y\right) \mid\left(R_{1}, R_{2}\right) \in W_{1} \times W_{2},\right. \\
\left.y=k\left(R_{1}, R_{2}\right) \underline{\Delta} k\left(v_{1}, v_{2}\right)\right\}
\end{gathered}
$$

Here

$W_{i}=\left\{R_{i} \mid R_{i}=\left(M_{i}, c_{i}, c_{i}\left(M_{i}\right)\right), c_{i}\left(M_{i}\right) \in V\left(c_{i}\right), i=1,2\right\}$, $V\left(c_{i}\right)$ is quantity territory of characteristic $c_{i}, k\left(v_{1}, v_{2}\right)$ is correlation function of corresponding in the extension relations $r$.

When $k\left(R_{1}, R_{2}\right) \leq 0$, to call $\sum$ for non-coordinating matter-element conjugate system, as $\Sigma-$. $O_{r}$ call $\Sigma$ for coordinating matter-element conjugate system, as $\sum+$. In particular:

Property 3:

$$
N_{1} \oplus L\left(N_{1}, N_{2}\right) \oplus N_{2}=\sum+
$$

Through property 2 and the meaning of correlation function, non-coordinating matter-element can be de- duced to be a conjugate system $\sum$ - which has contradiction waiting for solving. In-depth analysis principal aspect and the non-principal aspect of contradictory, primary reason causing system's non-coordinative can be found. If the non-coordinative of $\sum$ is mainly caused by the soft ministry sf $\sum$, we say $\sum$ for soft contradiction system, as $\sum-s f$; if the non-coordinative of $\Sigma$ mainly caused by the hard ministry hr $\sum$, we say $\sum$ for hard contradiction system, as $\sum-h r$. Non-coordinated element conjugated system is either the soft contradictory system, or the hard contradictory system.

Our goal is seeking the matter element transformation $T$, which transforms the non-coordinated matter-element conjugated system $\Sigma$ - to the coordinated matter-element conjugated system $\Sigma^{*}$. Namely seeking $T, T: \sum-\rightarrow$ $\Sigma^{*}$. Here, $\Sigma^{*}=N_{1}^{*} \odot L^{*}\left(N_{1}^{*}, N_{2}^{*}\right) \odot N_{2}^{*}$ is coordinated by the matter-element conjugated system. That is to say, for $R_{i}^{*}=\left(N_{i}^{*}, c_{j}, v_{j}\right),(i=1,2), R_{1}^{*}$ and $R_{2}^{*}$ are the relation of matter-element extension [5].

$$
\begin{gathered}
A^{*}(W)=\left\{\left(R_{1}^{*}, R_{2}^{*}, y\right) \mid\left(R_{1}^{*}, R_{2}^{*}\right) \in W_{1} \times W_{2},\right. \\
\left.y=k\left(R_{1}^{*}, R_{2}^{*}\right) \underline{\Delta} k\left(v_{1}, v_{2}\right)\right\}
\end{gathered}
$$

$k\left(v_{1}, v_{2}\right)$ is correlation function of corresponding extension relations $r^{*}$,and $k\left(R_{1}^{*}, R_{2}^{*}\right) \geq 0$.

\subsection{The Selection Principle and Conjugate Strategy of Matter-Element Transformation}

Since $\Sigma$ - is relatively close to realization of the system, to realize the $\Sigma$ - to $\Sigma^{*}$, we only can seek transform internal factors and transform way in inter system. The matter-element conjugation and matter-element transformation method provide a feasibility basis for this. In fact, the conjugate structure of $\sum$ directly leads to the transform principle of realizing the transformation $\Sigma$ to $\Sigma^{*}$ with the matter-element transformation method.

Principle 1: Desire for matter-element transformation $T$, preferred matter-element transformation of $T: \Sigma_{-} \rightarrow \Sigma^{*}$ is soft and hard transformation.

Principle 2: if $\sum-$ is soft contradiction system $\sum-s f$, desire for $T: \Sigma_{-} s f \rightarrow \Sigma^{*}$, We first choose initiative transformation $\varphi$ as the matter-element transformation to put on $s f \sum$; if $\sum-$ is hard contradiction system $\sum-h r$, desire for $T: \sum h r \rightarrow \Sigma^{*}$, We first choose initiative transformation $\varphi$ as the matter-element transformation to put on $h r \sum$.

Suppose initiative transformation $\varphi \rightarrow T_{\varphi}, T_{\varphi}$ is the conduction transformation caused by $\varphi$. The matter-element transformation in above principles can be expressed: $T_{h r}=\left(\varphi, T_{\varphi}\right), T_{s f}=\left(T_{\varphi}, \varphi\right)$.

As a result:

Principle 3: 


$$
T_{h r} \Sigma=\varphi(h r \Sigma) \oplus T_{\varphi}(s f \Sigma), T_{s f} \Sigma=T_{\varphi}(h r \Sigma) \oplus \varphi(s f \Sigma)
$$

Record $T_{h r}$ and $T_{s f}$ as $T^{*}$, when the initiative transformation $\varphi$ in $T^{*}$ is conjugate transformation, namely when $\varphi \in$ \{imaginary-real transformation, hard and soft transformation, appearance-latent Transformation, positive and negative transformation $\}$, call $T^{*}$ conjugate strategy.

Suppose $T^{*}$ is conjugate strategy, and $T^{*}: \Sigma_{-} \rightarrow \Sigma^{*}$, we call $T^{*}$ successful conjugate strategy, otherwise, $T^{*}$ is called unsuccessful conjugate strategy. All successful conjugate strategy constitute the successful conjugate strategy collection $L\left(T^{*}\right)$, correspondingly with coordinated matter-element conjugate system collection $\mathrm{L}$ $\left(\Sigma^{*}\right)$.Compare various strategies in strategy set $L\left(T^{*}\right)$, the optimal strategy as a final decision can be determined.

In applications, the implementation of conjugate transformation depends on conjugate converter. If you cannot construct suitable conjugate converter, $T^{*}$ is non-real strategy, no practical significance.

\subsection{Application of Extension Comprehensive Modeling System in Photosynthesis Process}

In limited matter element, particularly in the control system of D.huoshanense photosynthesis process, we have two ways to get process information: the data detected by process sensor and the experience of scene planting expert. But experts' experience is descriptive and is difficult or impossible to describe quantitatively (such as experts' experience which is perceivable but indescribable or confidentiality). The system modeling must use this part of experts' experience in the D.huoshanense photosynthesis process. Experts' experience is affected by many facts, it is very difficult to ensure experts' experience correctly reflecting the true nature of the process. This may cause workability process information to be sometimes serious shortage, sometimes squandered in D.huoshanense photosynthesis system modeling. We use the conjugate strategy to solve this problem.

Let be $N_{1}=$ extension modeling of D.huoshanense photosynthesis process, $N_{2}$ = experts' experience,

$L\left(N_{1}, N_{2}\right)=$ experience model, when other factors are not considered, relatively closed system:

$\sum=N_{1} \odot L\left(N_{1}, N_{2}\right) \odot N_{2}=\left(N_{1}, N_{2}\right) \oplus L\left(N_{1}, N_{2}\right)$ is a matter-element conjugated system. Examine the matter element $Q_{1}=\left(N_{1}, c_{1}, v_{1}(t)\right)$ and $Q_{2}=\left(N_{2}, c_{2}, v_{2}(t)\right)$.

Here $c_{1}=$ available information content in unit time, $v_{1}$ $=a$ (information entropy and constant $), c_{2}=$ the offer information in unit time, $v_{2}(t)$ is the function of time $t$, in the period of sufficient information, $v_{2}(t)>a$; in the period of lack information, $v_{2}(t)<a$.
For matter-element extension relations $A(W)$ of $Q_{1}$ and $Q_{2}$, correlation function $k\left(Q_{1}, Q_{2}\right)<0 . \quad \Sigma$ is non-coordinated matter-element conjugated system. Through close observation of the process of the system and environment of D.huoshanense photosynthesis process, the experience model of the system is found to get in long-term production practice. But when applied to D.huoshanense actual photosynthesis process, despite of large error with the actual process of photosynthesis, it contains rich of on-the-spot experts' experience information, specially has the quality experience information of D.huoshanenses. If matter-element transformation of extension theory can be used to blend expert experience model and the computer intelligent model, thus makes experts' experience that perceivable but indescribable or confidentiality give way to the scene plants the empirical model; in production field that expert is difficult to or is unable to accurate calculate or determination give way to the computer intelligence model. Then achieves the effect of "Cao Chong weighs elephant", hopefully get the innovation knowledge form it. Using this empirical model, in the period of sufficient information, the informative $v_{2}(t)-a$ as information power stores information (information changes from appearance to latency), while in the period of lack information, it can timely analyze its information (information changes from latency to appearance, to supplement information of the computer intelligence model when it lacks of information, and get a new information gain-using system) [4]. Therefore, choose the initiative transformation $\varphi=$ appearance-latency transforms, $\varphi \Rightarrow T_{\varphi}, T^{*}=\left(\varphi, T_{\varphi}\right)$, then

$$
T^{*} \sum=\varphi(h r \Sigma) \oplus T \varphi(s f \Sigma) \Rightarrow \Sigma^{*}=N_{1}^{*} L^{*}\left(N_{1}^{*}, N_{2}^{*}\right) \odot N_{2}^{*}
$$

Here, $\quad N_{1}^{*}=N_{1}, \quad N_{2}^{*}=N_{2}+N_{3} \quad\left(N_{3}\right.$ is empirical model), then $L^{*}\left(N_{1}^{*}, N_{2}^{*}\right)$ is new system.

Now, $Q_{1}^{*}=Q_{1}, Q_{2}^{*}=R_{21} \odot R_{22}, R_{21}=\left(N_{2}, c_{2}, v_{21}(t)\right)$, $R_{22}=\left(N_{3}, c_{2}, v_{22}(t)\right)$, and

$$
\begin{aligned}
& v_{21}(t)=\left\{\begin{array}{l}
a, t \in \Omega \\
v_{2}(t), t \in \Psi
\end{array}\right. \\
& v_{22}(t)=\left\{\begin{array}{l}
0, t \in \Omega \\
a-v_{2}(t), t \in \Psi
\end{array}\right.
\end{aligned}
$$

where $\Omega=$ \{period of sufficient $\}, \Psi=$ \{period of lack $\}$.

For matter-element extension relations $A^{*}(W)$ of $Q_{1}^{*}$ and $Q_{2}^{*}$, correlation function $k\left(Q_{1}^{*}, Q_{2}^{*}\right) \geq 0, \Sigma^{*}$ is non-coordinated element conjugated system. The conjugate converter may take:

It is difficult or impossible to describe experts' experience gain $\rightarrow$ empirical model $\rightarrow$ use of experts' experience, therefore $T^{*}$ is a successful conjugate strategy. 


\section{Matter-Element Extension Transformation Method of Modeling Strategy}

According to the request of modeling strategy in D.huoshanense photosynthesis process, determine purpose matter-element of D.huoshanense:

$$
W=\{R \mid R=(N, c, v), N \in U, v \in V\} .
$$

And appropriate correlation function, $y=K(v)$, $v \in V, y \in(-\infty,+\infty)$, establishes the matter-element extension set in $W$ :

$$
A(R)=\{(R, y) \mid y=K(R)=K(v)\}
$$

Production strategy, also can say seeking transformation $T$, makes negative field matter-element $R(K(R) \leq 0)$ transform into positive field matter-element $(K(R) \geq 0)$. That is to say determine transformation $T$ which can determine $A_{+}(R)(T)$, seek transformation $T_{R}, T_{K}, T_{W}$ from three angles that matter-element $R$, correlation function $K$ and the universe of discourse $W$ to formation positive extension field of matter-element:

$$
\left\{\begin{array}{l}
A_{+}(R)\left(T_{R}\right)=\left\{R \mid R \in W, K(R) \leq 0, K\left(T_{R} R\right) \geq 0\right\} \\
A_{+}(R)\left(T_{K}\right)=\left\{R \mid R \in W, K(R) \leq 0, K\left(T_{K} R\right) \geq 0\right\} \\
A_{+}(R)\left(T_{W}\right)=\left\{R \mid R \in W, K(R) \leq 0, R \in T_{W}(W), K(R) \geq 0\right\}
\end{array}\right.
$$

According to the current research of the extension theory, we can make choice mainly in displacement transformation, additions and deletions transformation, extend and reduce transformation, decomposition transformation, transition transformation, catalytic transformation and recover loss transformation, also can consists transformation combination $T=\left\{T_{i} \mid i=1,2, \cdots, k\right\}$ using four operations (product, and, or, inverse) of transformation [1].

Start with method of generating strategy set from extension matter-element extension field, mainly aimed at incompatibility issues of target and conditions during modeling strategy, we can exploit more practical modeling strategies to meet the needs of the right strategies using extension analysis method.

\subsection{Transforming Bridge Method of Modeling Strategy}

Opposition problems (the same conditions contradiction between two groups target strategy under the same conditions) can be seen everywhere in real-life .In the conflict of the area, often use the struggle method (It may cause new conflict), or use balance method (can defer outbreak of conflict). If choosing transform bridge method, setting appropriate transforming bridge, we transform each index system (or operation rules) of contradiction target form opposition system into coexisting in combination areas. That is a new method of formalization of creative thinking process .Microclimate environment of greenhouse has three stages of control strategy to planting process of D.huoshanense: switch control strategy of the stage transplanting seedling of D.huoshanense to field, predictive control and feed-forward control strategy of survival stage of seedling, tracking control strategy at stable growth phase. The third groups of strategy are in the same microclimate environment of greenhouse, but the goal of three strategies under the condition is different. In the conflict the area, setting appropriate transforming bridge, we transform index system (or operation rules) of three strategies goals into coexisting in combination areas. We make them exist in greenhouse microclimate environment, and achieved the effect that operates separately, takes their want.

First discusses two group of opposition strategies [4]: let be two opposing strategy targets as system $L_{1}$ and system $L_{2}$, index system is expressed as multidimensional dynamic matter-element $S_{1}(d, t)$ and $S_{2}(d, t), M=$ $\left(S_{1}(d, t), S_{2}(d, t)\right)$, then $S_{1}$ and $S_{2}$ is a opposing system of $M:\left(L_{1} \uparrow L_{2}, M\right)$.

Setting combination areas $A_{i}\left(L_{1}, L_{2}\right),(i=1,2, \cdots, k)$ in the conflict the area of $L_{1}$ and $L_{2}$, establishing: $L=$ $L_{1} \odot A_{i}\left(L_{1}, L_{2}\right) \odot L_{2}$, Index system of system:

$$
S(d, t)=\left\{\begin{array}{l}
S_{1}(d, t) \text { in } L_{1} ; \\
S_{1}(d, t) \wedge S_{2}(d, t) \quad \text { on } A_{i}\left(L_{1}, L_{2}\right) \\
S_{2}(d, t) \text { in } L_{2} .
\end{array}\right.
$$

$L$ is compatible system of $L_{1}$ and $L_{2}$ which is a opposing system of $M, A_{i}\left(L_{1}, L_{2}\right)(i=1,2, \cdots, k)$ is the transforming bridge of $L_{1}$ and $L_{2}$.

Let be $A_{i}\left(L_{1}, L_{2}\right)=\left(O_{1}\right.$ and $O_{2}$ respectively are movement track of $L_{1}$ and $\left.L_{2}\right)$,index system of $A_{i}\left(L_{1}, L_{2}\right)$ is

$$
S(d, t)=S_{1}(d, t) \wedge S_{2}(d, t)=\left\{\begin{array}{lll}
S_{1}(d, t) & \text { on } & L_{1} \\
S_{2}(d, t) & \text { on } & L_{2}
\end{array}\right.
$$

On the transforming bridge $A_{i}\left(L_{1}, L_{2}\right)$, movement track is $O=O_{1} \odot O_{2}$, we usual use space passage $k(d)$ or time channel $k(t)$ to insulate $O_{1}$ and $O_{2}$.

Then discusses three group of opposition strategies: let be three opposing strategy targets as system $L_{1}$, system $L_{2}$ and system $L_{3}$, index system is expressed as multidimensional dynamic matter-element $S_{1}(d, t), S_{2}(d, t)$, and $S_{3}(d, t)$, then $S_{1}, S_{2}$ and $S_{3}$ is a opposing system of $M$ : $\left(L_{1} \uparrow L_{2} \uparrow L_{3}, M\right)$.

Setting combination areas $A_{i j}\left(L_{1}, L_{2}, L_{3}\right),(i=1,2, \cdots$, $k, j=1,2,3)$ in the conflict the area of $L_{1}, L_{2}$ and $L_{3}$. establishing: $L=L_{1} \odot A_{i 1}\left(L_{1}, L_{2}\right) \odot L_{2} \odot \mathrm{A}_{\mathrm{i} 2}\left(L_{2}, L_{3}\right) \odot L_{3} \odot$ $A_{i 3}\left(L_{2}, L_{3}\right) \odot L_{1}$, index system of system: 


$$
S(d, t)=\left\{\begin{array}{lll}
S_{1}(d, t) \quad \text { in } \quad L_{1} ; & \\
S_{1}(d, t) \wedge S_{2}(d, t) & \text { on } & A_{i 1}\left(L_{1}, L_{2}\right) ; \\
S_{2}(d, t) \wedge S_{3}(d, t) & \text { on } & A_{i 2}\left(L_{2}, L_{3}\right) \\
S_{3}(d, t) \wedge S_{1}(d, t) & \text { on } & A_{i 3}\left(L_{3}, L_{1}\right) ; \\
S_{2}(d, t) & \text { in } L_{2} ; & \\
S_{3}(d, t) \quad \text { in } L_{3} ;
\end{array}\right.
$$

$L$ is compatible system of $L_{1}, L_{2}$ and $L_{3}$ which is a opposing system of $M, A_{i j}\left(L_{1}, L_{2}, L_{3}\right)(i=1,2, \cdots, \mathrm{k}, j=1$, $2,3)$ is the transforming bridge of $L_{1}, L_{2}$ and $L_{3}$.

Let be $A_{i j}\left(L_{1}, L_{2}, L_{3}\right)=\left(O_{1}, O_{2}\right.$ and $O_{3}$ respectively are movement track of $L_{1}, L_{2}$ and $\left.L_{3}\right)$, index system of $A_{i}\left(L_{1}\right.$, $\left.L_{2}, L_{3}\right)$ is:

$$
\begin{aligned}
S(d, t) & =S_{1}(d, t) \wedge S_{2}(d, t) \wedge S_{3}(d, t) \\
& =\left\{\begin{array}{lll}
S_{1}(d, t) & \text { on } & L_{1} ; \\
S_{2}(d, t) & \text { on } & L_{2} ; \\
S_{3}(d, t) & \text { on } & L_{3} ;
\end{array}\right.
\end{aligned}
$$

On the transforming bridge $A_{i j}\left(L_{1}, L_{2}, L_{3}\right)$, movement track is $O=O_{1} \odot O_{2} \odot \mathrm{O}_{3}$, we usual use space passage $k(d)$ or time channel $k(t)$ to insulate $O_{1}, O_{2}$ and $O_{3}$.

\subsection{Evaluation and Selection of Modeling Strategies}

We should value each strategy in D.huoshanense photosynthesis process strategy set, and chose a better strategy for use. We can take superiority evaluation method in the process of evaluation and selection, (see Figure 1) [6]:

1) According to technical indexes of D.huoshanense photosynthesis process control, the economic value and quality determine of Chinese medicine experts, we can ensure conditions set which is used to measure: $r=\left\{r_{1}\right.$, $\left.r_{2}, \cdots, r_{n}\right\}, r_{i}=\left(c_{i}, v_{i}\right), v_{i}$ is quantity range, $i=1,2, \cdots, n$.

2) Selecting the conditions satisfy with D.huoshanense photosynthesis process, as $m=\left\{m_{1}, m_{2}, \cdots, m_{s}\right\}$, we uses it to sift concentrate strategy in strategy set; then bestow the weighting factor between $[0,1]$ to the rest measure condition according to its important degree: $\alpha=$ $\left\{\alpha_{1}, \alpha_{2}, \cdots, \alpha_{n}\right\}, \sum \alpha_{i}=1$.

According to the condition $\alpha$, through primary selection of produces strategy, the strategy accord with the requirement for $\alpha$ is: $U_{j}=\left\{U_{j} \mid j=1,2, \cdots, m\right\}$.

3) Establishing correlation function $k_{i}(x)$ on $V_{i}$, calculating standard qualified degree of $U_{j}$ respect to $r_{i}: k_{i}=\left(r_{i 1}\right.$, $\left.r_{i 2}, \cdots, r_{i m}\right), i=1,2, \cdots, n$.

4) Computation goodness of fit:

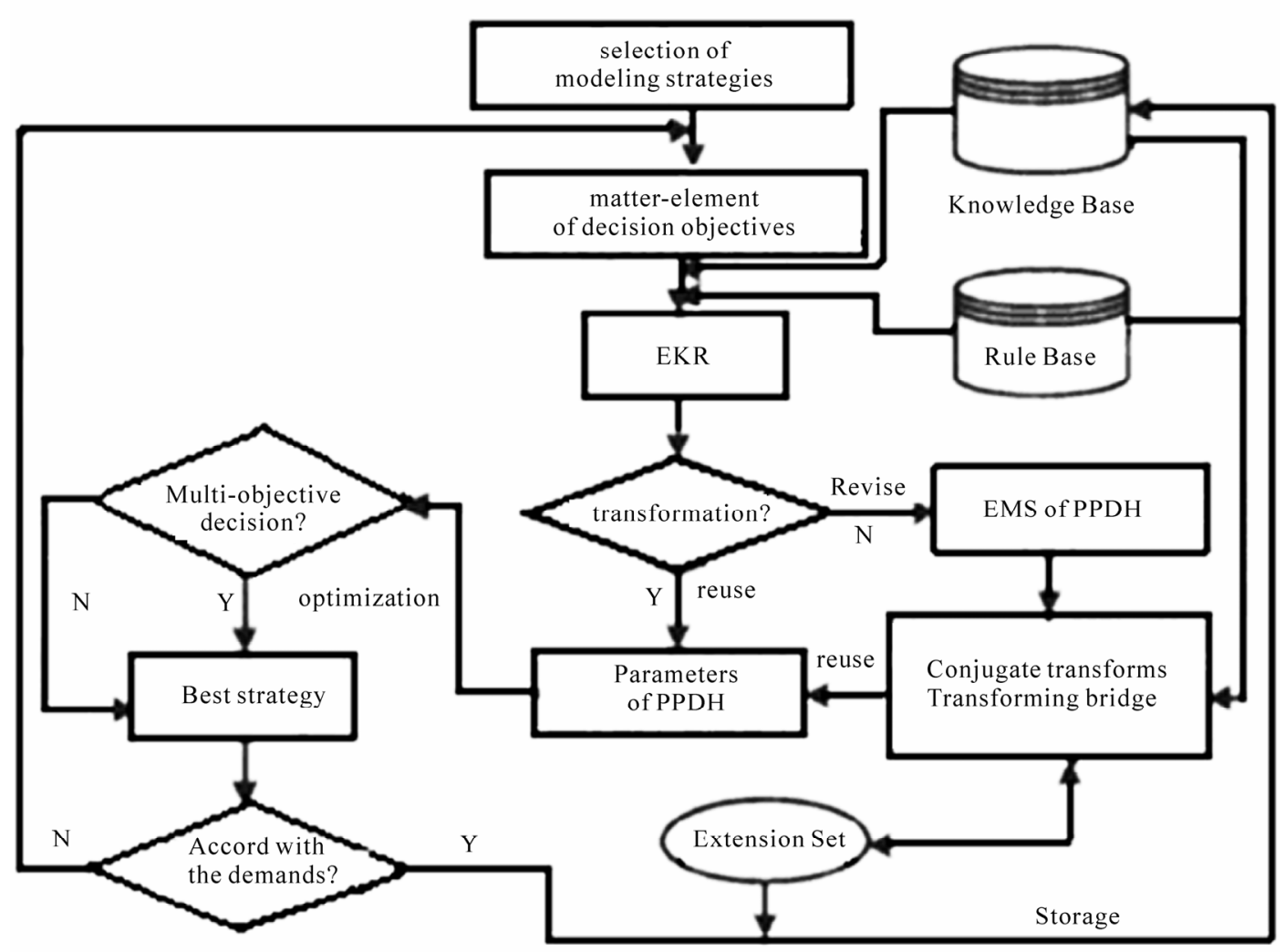

Figure 1. The extension solving model (ESM) based on EKR [6]. 


$$
B\left[U_{j}\right]=\sum_{i=1}^{n} \alpha_{i} k_{i j}, j=1,2, \cdots, m .
$$

Best strategy:

$$
B\left(U_{0}\right)=\max \left\{B\left(U_{j}\right) \mid j=1,2, \cdots, m\right\}
$$

\section{Conclusions}

Since the limitations of the existing knowledge representations in intelligent detection, this article proposes a new representation based on multi-level Extension Modeling Strategy and develops a corresponding detection system of D.huoshanense photosynthesis process, which has positively be carried on the new exploration to the intellectualized modeling strategy. Compared with the previous modeling strategies, choosing different selection size according to features of different growth phase, this method not only can avoid the phenomenon that the explosion of elements reorganization in modeling strategies, still can make the innovation of new strategy set in higher levels. At the same time, with the formalized inference technology of extension theory, combining with quantitatively reasoning, and it successful realize the transition between concept model and detailed model of detection strategy in D.huoshanense photosynthesis process. Further work is research algorithm complexity of POADES (Problem Oriented Analysis and Decision Expert System) [7]; Increases the structure operator on the basic of POADES to strengthen the ability that description model concept or relational, make the description logic be adapted to the needs of various applications.

\section{Acknowledgements}

This work has been supported by Financed project of NSFC, under 60974038 grants.

\section{References}

[1] W. Cai, C. Y. Yang and G. H. Wang, "A New Cross Subject-Extenics," Bulletin of National Nature Science Foundation of China, in Chinese, Vol. 18, No. 5, 2004, pp. 268-272.

[2] S.-S. Zhong and Y. Zhang, "Extension-Based Knowledge Representation and Development of Knowledge Base System," Computer Integrated Manufacturing Systems, in Chinese, Vol. 14, No. 11, 2008, pp. 2184-2190.

[3] L. X. Li and J. Li, "Extension Knowledge Base System and Its Application," Engineering Science, in Chinese, Vol. 3, No. 3, 2001, pp. 61-64.

[4] R. D. Lu and Z. H. Cheng, "Matter-Element Modeling of Parallel Structure and Application about Extension PID Control System," Journal of Systems Science and Complexity, Vol. 19, No. 1, 2006, pp. 227-235. doi:10.1007/s11424-006-0227-9

[5] W. Cai, C. Y. Yang and B. He, "Basic Extension Logic," Science Publishers, Beijing, 2003.

[6] Z. G. Nian, et al., "Study and Application of Knowledge Expression," Application Research of Computers, in Chinese, Vol. 24, No. 5, 2007, pp. 234-246.

[7] R. D. Lu, X. P. Tao, Q. Sun and Y. X. Ren, "Detection in Biological and Pharmacological Characteristics and Photosynthesis Process-A Key Point and Direction in Dendrobium Huoshanense Study," Chinese Traditional and Herbal Drugs, in Chinese, Vol. 41, No. 11, 2010, 1917 1920. 